\title{
Liquid Phase Epitaxy for Optical Communication Devices
}

\author{
Mohammad Asadul Haque, Zahid Hasan Mahmood and Jalalur Rahman
}

Department of Applied Physics, Electronics and Communication Engineering, University of Dhaka, Dhaka-1000, Bangladesh

\begin{abstract}
In this review paper we discuss the scope of the Liquid Phase Epitaxy (LPE) for the growth of the semiconductor materials required for optical communication devices. LPE is non-hazardous comparatively cheaper technology for the material growth and growth processing laboratory could be set up easily. LPE offers the selective and equilibrium growth which is suitable for simple structures. LPE set up could be easily installed for the research and academic institutes as well as in the industries.
\end{abstract}

Key words: Liquid Phase Epitaxy (LPE), Impurities, Optical Communications, Multilayer, Heterostructures, Light Emitting Diodes (LED), Photodetectors, Solar cells and Photodiodes.

\section{Introduction}

Semiconductor devices are presently used in almost all branches of electronics including communication Engineering. These devices can be grown by different growth technology namely VPE, MOCVD, MBE, LPE etc. Liquid Phase Epitaxy (LPE) is simple and cost effective method to fabricate semiconductor devices. It is a technique in which the epitaxial layer grows from solutions at elevated temperatures over a single crystal substrate. The basis of Liquid Phase Epitaxy is the control of liquid-solid phase equilibrium. It is an equilibrium growth process, in which the defects are minimum. The growth rate is high and thick layers can be grown very easily. The success of LPE growth depends on temperature control, solution composition and substrate preparation. There are a number of subsidiary factors which affect the layer quality such as ambient gas purity, the design of the growth equipments (loading system, furnaces), the method of separation of the solution from the growth layer and the geometry of the growth solution (Astles M. G., 1990).

\section{Basic Information on LPE}

Liquid Phase Epitaxy (LPE) is a technique by which thin epitaxial layers of semiconductors are grown over semiconductor substrate from supersaturated solutions. The epitaxial layer is often chemically more pure than the original source semiconductor, due to very low values of the segregation coefficient for most of the common impurities. The LPE tech-

\footnotetext{
* Corresponding author: E-mail: asad2310@yahoo.com
}

nique was first demonstrated by R.J. Nelson of Bell Laboratories in 1963 by fabricating Ge tunnel diodes and GaAs lasers (Alexiev D. et al. 2006). Till that date the use of LPE has grown very rapidly. Today the use of LPE has mainly concentrated on the growth of III-V compound semiconductors and its ternary and quaternary alloys.

The process of growth may be understood by considering the phase diagram of the GaAs system Fig. -1. Solid GaAs can exist in equilibrium with a range of liquidus compositions.

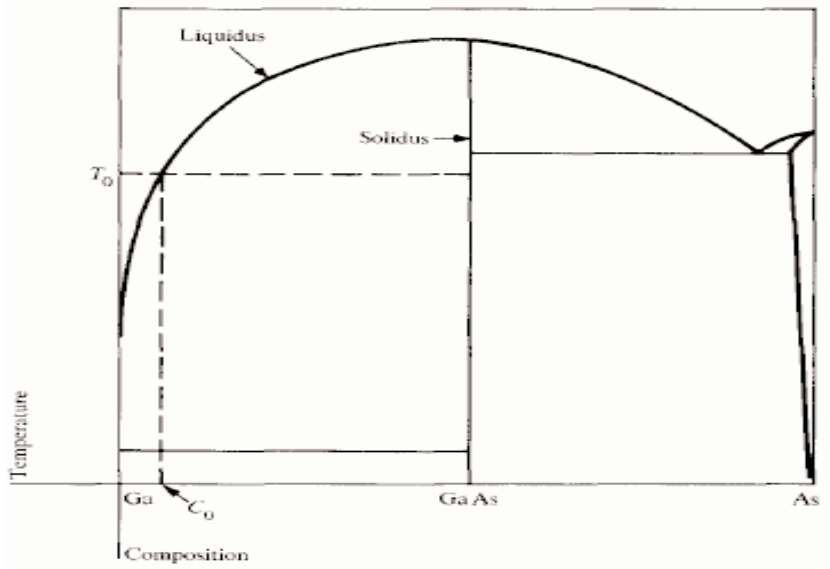

Fig. 1. Phase diagram of the GaAs system (Cowen J. et al. 2005)

Liquid Phase Epitaxy technique is widely used for the growth of composite semiconductor layer structures for the manufacture of optoelectronic devices. 


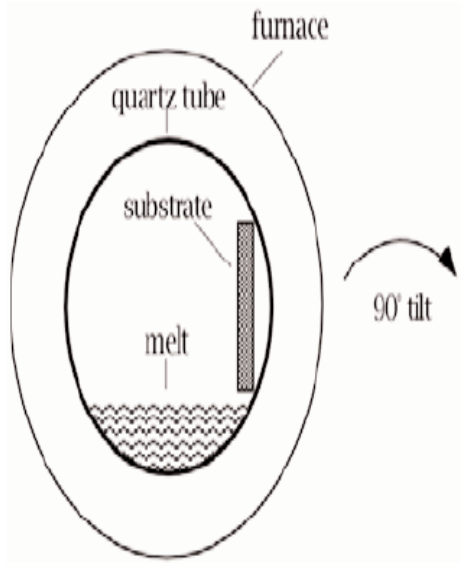

(a)

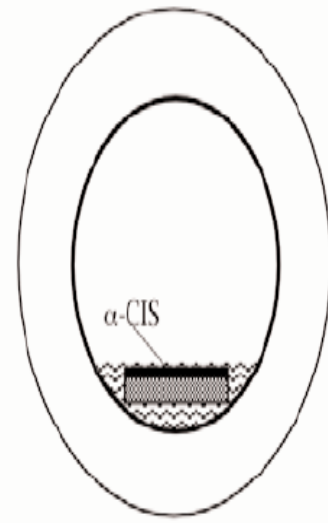

(b)
Fig. 2. The concept of liquid-phase deposition (LPD).

(a) CuInSe2melt with a composition in one of the shaded fields

(b) Deposition of a thin $\alpha$-CuInSe2 film induced by slightly super cooling the melt in contact with a substrate (Ernst F. et al. 2006).

LPE has some distinct advantages that are:

1. It is a simple process that is suitable for selective growth.

2. It is an equilibrium growth process that can produce high purity materials.

3. It is inexpensive, rather non-hazardus and highly suitable for simple structures.

4. It offers controlled $n$-type or $p$-type doping with a variety of dopents available.

5. The epitaxial layers are often chemically more pure than the original source semiconductor, due to very low values of the segregation co-efficient for most of the common impurities.

6. The alloy composition of ternary and quaternary compounds is determined by precise weighting the components in the melt and by establishing the growth temperature.

These are easily reproducible producers and easier to control. However this growth process is unsuitable for the growth of thin layers and sharp interfaces and is not suitable where extremely accurate layer thickness uniformity and reproducibility are required.

\section{Sources of impurities in LPE growth and protection}

During the growth process, several kinds of metallic and non metallic impurities may be introduced in the grown layer from the following possible sources (Dhar S., 2005):

a) the source materials themselves and the chemicals or reagents used to clean them

b) the walls of the growth boat which comes in contact with the melt

c) contaminants deposited on the inner walls of the quartz reactor tube which are transported to the melt by the ambient gas, during high temperature growth

d) the carrier hydrogen gas itself and the impurities carried by the gas from the inner walls of the gas lines

e) the containers for storing, handling and cleaning of the materials and substrate.

In order to minimize the impurities coming from the above sources the materials for growth should be taken in high purity form, the LPE growth boat should be made of high purity low porosity graphite which will helps to keep the absorbed gasses to a minimum, the reactor tube should be made of high purity quartz and before every use the inside of the tube should be cleaned and etched in HF based reagents, Pd-diffused hydrogen gas is used as the growth ambient and pyrex or corning glass containers may be used for cleaning the source materials and the substrate.

\section{Advantages in LPE over other growth technologies:}

Semiconductor multilayer heterostructures may be grown by a number of epitaxial processes: liquid phase Epitaxy (LPE), molecular beam epitaxy (MBE) or chemical vapor deposition (CVD). For the growth of lasers LPE is at present the most commonly used process.

One reason for this is that material grown by the LPE process generally has a higher luminescent efficiency than that produced by the other processes (Lynch R.T., 2004). Another reason is that (GaAl)As cannot be grown by the conventional halide process for CVD because of the reactivity of AI with oxygen, which causes the AI vapor to react with the glass envelope (Lynch R.T., 2004). LPE remains at present the main method for laser fabrication. Our discussion is particularly directed toward procedures and equipment which provide good control over the compositions, dimensions, and crystal quality of the material grown.

\subsection{Compositional considerations}

Liquid phase epitaxy is a solution growth process in which a series of layers of defined compositions could be grown from a sequence of cooling solutions in a single operation. 
The process of growth might be understood by considering the phase diagram of the GaAs system (Fig. 1). Solid GaAs can exist in equilibrium with a range of liquidus compositions.

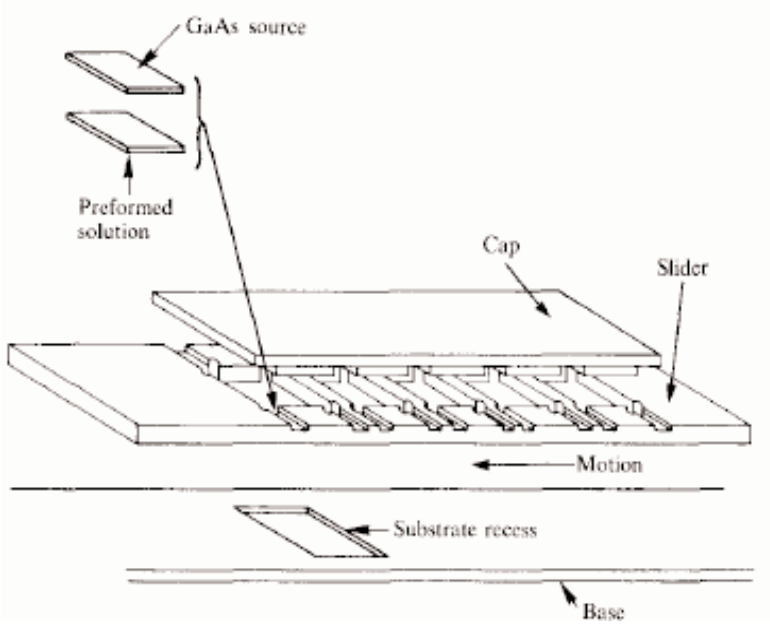

Fig. 3. Schematic view of the growth crucible (Lynch R.T, 2004)

\subsection{Crystal quality}

Crystal quality is important for a good yield of long-lived lasers. Dislocations threading through the active layer of a device lead to the development of large networks of dislocations during the operation and subsequent degradation of the device. Some of these dislocations originate in the substrate. Substrates are obtainable with a sufficiently low density of these defects to provide a high yield of long-lived lasers. During the epitaxial growth process it is important not to produce a greater density of dislocations than that present in the substrate; this might occur if solid impurity particles are included in the growing layer.

\section{LPE grown III-V semiconductors}

The compound semiconductors like GaAs, AlAs, GaN, InP, GaAsSb etc could be grown by LPE. The LPE materials are on the basis of optoelectronic devices, so it should be consider the band gap as an optical parameter. The internal luminescent efficiency of LPE grown layers of III-V binaries is normally high due to the low concentrations of deep-level carrier traps, low dislocation densities and uniformity of the layers. For controlling the layer thickness there are four primary techniques of LPE which is based on the sliding boat apparatus and reproducibility of the system. The ability to grow abrupt heterojunctions or doped homojunctions is an important fact of any epitaxial growth technology. In case of LPE this is achieved by having a variety of growth solutions in the growth system. The abruptness of the junctions grown by LPE is mainly determined by several factors such as substrate instability, transient growth, cross contamination problems and diffusion (Astles M. G., 1990).

\section{LPE for optoelectronic devices and optical commu- nications}

The LPE grown optoelectronic devices includes LED, LASERS, photo-detectors, optoelectronic integrated circuits, solar cells and many others. These optoelectronic devices play a significant role in the optical communication technology.

\subsection{Light emitting diodes (LEDs)}

One of the earliest applications of LPE was to the growth of materials for LEDs, initially in the visible part of the spectrum for display applications.

The device utilizes the electroluminescence produced when minority carriers injected across a $\mathrm{p}-\mathrm{n}$ junction recombine, emitting light of a wave length which normally corresponds closely to the band gap. For the increment of the external quantum efficiency of LED, Tsai and Wu (Tsai M. J. et al. 1996) have described a high-brightness red LED using the diffused and double epitaxy LED structures providing low recombination velocity interface, increased injection efficiency of minority carriers into the active layer, a transparent top layer. The developments of LPE products such as LED, plasma CRT and electroluminescent displays plays a significant role in case of domestic products such as calculators, wrist watch and other equipments where low power consumption is essential. Recently, attention has been focused on the LPE growth of high brightness red AlGaAs LEDs for use in automobile tail lights. Today the most important application of high efficiency LED lie in the area of silica fiber based optical communications.

\subsection{Applications of Magneto-optical Garnet Layers}

Magnetic garnets represent a class of materials with outstanding importance in electronic devices (Aichele T. et al. 2003).

The garnet LPE is already somewhat like a model system for liquid phase epitaxy and flux growth respectively. The mag- 
netic field sensitivity of magneto-optical garnet films is high enough to support a number of applications for magnetic field detection. In particular, the excellent area homogeneity of garnet LPE films provides the basis for imaging of magnetic fields with high lateral resolution. Technical targets for application are magnetic field sensors, current sensors, magnetic field imaging, and non-destructive evaluation (NDE). Principle components of a magneto-optical field sensing device are an intense source of plane polarised light, the magneto-optical garnet film being placed in the magnetic field to be measured and a detection unit for probing the polarisation state after Faraday rotation. The commonly applied transmitting mode of operation of a Faraday rotator may be converted into a reflection mode by providing the garnet film with a reflection layer. In this case the sensor may be placed in front of the surface of a magnetic material or device to image its stray field distribution. The light beam crosses twice the garnet layer doubling the rotation angle in this way. The high quality of LPE grown garnet films allows observing the stray field with high spatial resolution using an optical microscope with large magnification. The principal set-up of a magneto-optical imaging device is schematically shown in Fig. 4.

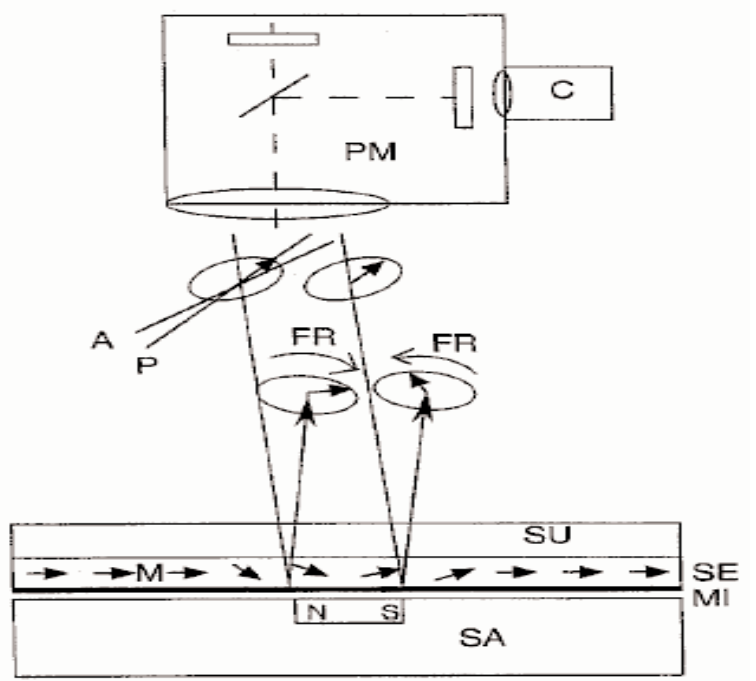

Fig. 4: Principal setup of a magneto-optical microscope (schematically). (Aichele T. et al. 2003)

The most important of which are optical noise originating in the illumination system (laser), electronic noise due to the detection system, and the media noise due to the magnetooptical garnet film. The latter one may principally originate from magnetisation processes involving domain motion, from garnet film defects, and from surface roughness of the sensor films. The above discussed applications concentrate on garnet films with in-plane anisotropy.

\subsection{Semiconductor lasers}

Applications that use semiconductor lasers as heat sources generally must operate at fairly high optical power compared to that used in optical communications systems.

Writing on a video disk is usually done with very high peak power, short pulses. Liquid crystal displays cells could be require tens of milliwatts of laser output for reasonable writing speeds. Reliability under high-power pulse operation is mostly concerned with sudden catastrophic damage to the laser facets resulting from the very large optical field strengths.

Semiconductor lasers are preferred to LEDs as light sources for communication because of their coherence, stronger irradiance and ability to get better coupling with optical fibers. The targets for better lasers, other than achieving the desired wavelength regions are lowering of the threshold current and continuous operation at room temperature. The semiconductors laser works, by analogy with its solid state or gas counterparts, by the stimulated emission of coherent radiation brought about by population inversion. Since then for the development of laser structures scientists (Ernst F. et al. 2006, Aichele T. et al. 2003) have had several aims such as reduction in junction width Jth,, Increasing lifetime, control of laser mode structure etc.

Reduction in junction width Jth could be achieved by producing carrier and optical confinement in the active layer by cladding it with layers with highest bandgap and lower refractive index. Reductions in Jth could also be achieved by reducing the active layer thickness and by increasing the quantum efficiency could be possible by the improvements in the LPE growth process in terms of growth rate control and defect deduction.

Dislocations in the epitaxial layers could be

i) caused by lattice mismatch between the layer and substrate.

ii) propagated from the substrate.

iii) introduced during device fabrication.

The control of the longitudinal and transverse modes of the laser is extremely important in order to

a) obtain efficient coupling of the laser output into the- 
monomode fibers used in modern high bit-rate, long haul fiber-optic communications systems.

b) minimize dispersion effects in these fibers

c) provide the stable mode behavior required in several applications such as laser printing or optical disc recording/ playback systems and

d) provide wavelength stability.

\subsection{Photodetectors}

Fabrication of photoconductors is a promising area where LPE could be successfully used and the material requirements are best met by this technique. There are several categories of photodetector which differ in the way in which the charge is detected. The principle categories are as follows:

\section{4-1 Photodiodes}

The charge is controlled by a p-n junction. If the diode is operated at high reverse bias, the photo-induced charge carriers are accelerated and can release other carriers producing a current again. This device is called avalanche photodiode. The key parameters of a photodiode used as a receiver in optical communications systems in conjunction with typically GaAs MESFET amplifier are a) high quantum efficiency b) fast response time c) low noise d) low dark current e) uniform gain in the case of avalanche photodiodes.

\section{4-2 Photocathodes}

Scheer et al (Scheer J. J. et al. 1965) and Turbull et al (Turnbull A A et al. 1968) demonstrated the potential of producing a negative electron affinity photocathode imaging tube with single crystal GaAs materials. It can improve spatial resolution due to the low spread of transverse electron energies. A high minority carrier diffusion length and low recombination velocity at the interface are responsible for the photocathode efficiency.

\section{4-3 Phototransistors}

The carriers produce a change in the conductance of the transistors. These devices could be incorporated in more complex integrated device structures such as optoelectronic integrated circuits (OEIC). The benefits of such integration circuits are chiefly a) higher operating speed due to reduced parasitic impedance, b) improved reliability and c) reduced production costs. Sophisticated optoelectronic integrated circuits have been successfully grown in Japan by LPE.

\subsection{Solar cells}

Based on the expected structural quality of the material, it could be anticipated substantial improvements in photovoltaic conversion efficiency. Moreover, the comparison between the anticipated, coarse-grained LPD material and fine-grained PVD material could be supposed to provide deeper insight into the effect of grain boundaries on photovoltaic conversion in a-CuInSe2, for which a new model was recently proposed (Ernst F. et al. 2006).

Reduction of cost of solar cell is a need recognized globally. As the cost of amount of electronic grade silicon wafer used in a solar cell is a substantial fraction of its total cost, thin silicon solar cell fabricated on low cost substrates by an inexpensive method called LPE. LPE facilities auto-doping of thin epitaxial layer and thereby introduces drift field in solar cell without any extra step (Bergmann R., 1993). The LPE layer on highly doped Si-substrate produces back surface field which could help to increase the efficiency of the solar cell (Zheng G. F. et al. 1996). Peter et al (Peter K, et al. 2002) mentioned on LPE grown layer silicon it could be possible $19 \%$ efficiency.

The field of solar photovoltaic energy conversion has been an extremely active in the possibility of large-scale solar power stations to provide local domestic and industrial electric power for isolated communities using large areas of very cheap solar cell and the provision of solar power for the satellites increasingly used for telecommunications, surveillance, resource mapping, integrated circuits (OEIC). The benefits of such integration circuits are chiefly a) higher operating speed due to reduced parasitic impedance, b) improved reliability and c) reduced production costs. Sophisticated optoelectronic integrated circuits have been successfully grown in Japan by LPE.

\subsection{Solar cells}

Based on the expected structural quality of the material, it could be anticipated substantial improvements in photovoltaic conversion efficiency. Moreover, the comparison between the anticipated, coarse-grained LPD material and fine-grained PVD material could be supposed to provide deeper insight into the effect of grain boundaries on photovoltaic conversion in a-CuInSe2, for which a new model was recently proposed (Ernst F. et al. 2006).

Reduction of cost of solar cell is a need recognized globally. As the cost of amount of electronic grade silicon wafer used 
in a solar cell is a substantial fraction of its total cost, thin silicon solar cell fabricated on low cost substrates by an inexpensive method called LPE. LPE facilities auto-doping of thin epitaxial layer and thereby introduces drift field in solar cell without any extra step (Bergmann R., 1993). The LPE layer on highly doped Si-substrate produces back surface field which could help to increase the efficiency of the solar cell (Zheng G. F. et al. 1996). Peter et al (Peter K, et al. 2002) mentioned on LPE grown layer silicon it could be possible 19\% efficiency.

The field of solar photovoltaic energy conversion has been an extremely active in the possibility of large-scale solar power stations to provide local domestic and industrial electric power for isolated communities using large areas of very cheap solar cell and the provision of solar power for the satellites increasingly used for telecommunications, surveillance, resource mapping, meteorological and space laboratory applications (Majumder D. et al. 2003).

\section{Conclusion}

It is now well-established that LPE in its current form is not the technology for the production of sophisticated devices involving complex structures with carefully engineered energy band profiles. It may also be a viable technique for the commercial manufacture of high speed transistors, FETs, Gunn diodes, IMPATTS where, MOCVD because of its batch production capability has a definite edge. However there appears to be some secured areas for LPE materials where the devices have simple structures but at the same time demand high quality material produced by a less sophisticated technology.

\section{Scope for future work}

The success of LPE growth depends on a large number of factors. Apart from the dominant ones of temperature control, solution composition and substrate preparation, there are a number of subsidiary factors which affects the layer quality and yield, such as the ambient gas purity, the design of the growth equipment (loading systems, furnace), the method of separation of the solution from the growth layer and geometry of the growth solution. Developments in the area of automatic control, boat and crucible design, substrate preparation, supersaturation, lower temperature, ultrapure metals polycrystalline semiconductors, etc of LPE technology which could lead to enhancements in material quality.

\section{References}

ASTLES M. G. and Adam Hilger I. O .P publications, (1990) Liquid phase epitaxial growth of III-V compound.
ASTES M. G., Adam Hilger and I. O. P publications, (1990) Semiconductor materials and their device applications

Aichele T., Lorenz A., Hergt R. and Görnert P. (2003) Garnet layers prepared by liquid phase epitaxy for microwave and magneto-optical applications a review Cryst. Res. Technol. 38 (7-8): 575 - 587.

Alexiev D., Prokopovish D. A., Thomson S. and Reinhard M. (2006) A review of liquid phase epitaxial grown Gallium Arsenide, a report on LPE at the Austalian Nuclear Science and Technology Organization.

Bergmann R and Kurianski (1993) J Mater, Letts. 17: 137143.

Cowen J., Lucas L., Ernst F., Pirouz P., Hepp A. and Bailey S. (2005) Liquid-phase deposition of single-phase alpha-copper-indium- diselenide Materials Science and Engineering, 116: 311-319.

Dhar S. (2005) Growth of high purity semiconductor epitaxial layers by liquid phase Epitaxy and their characterization Indian academy of Science, 28(4): 349-365.

Ernst F. and Pirouz P. ( 2006) Liquid- Phase Deposition of CIS Thin Layers Final report, NREL/SR-520-39341.

Lynch R. T., Jr. Small M. B. and Hung R. Y. (2004) GaAsl (GaAl)As Laser Technology IBM 1. res. develop. 23 (5): 365-369.

Majumder D., Chatterjee S. and Saha H. (2003) Modified technique of using conventional slider boat for liquid phase Epitaxy of silicon for solar cell application Bull. Mater. Sci. 26: 643- 646.

Peter K., Kopecek R, Hotzel Fath P., Butcher E., Zahedi C. and Farrazza F. (2002) Sol. Energy Mater and Sol. Cells 74: 219-224.

Scheer J. J. and Vanlaar J. (1965) Solid-state commun 3: 189-192.

Tsai M. J. and Wu C. H. (1996) 11th Int. Symp. On GaAs and Related Compounds (Biarritz) (Inst. Phys.Conf. Ser. No. 74: 439-456.

Turnbull A. A. and Evans G. B. (1968) J Phys. D. Appl. Phys. 1: 155-175.

Zheng G. F., Zhang W, Shi Z, Sproul A. B., Gross M. and Green M . A, (1996) Sol. Energy Matter. and Sol. Cells 40: 231-146.

Received : August, 13, 2008;

Accepted : February 11, 2009 
